NASA Technical Memorandum 105363

AIAA-92-0794

\title{
Synergistic Effects of Ultraviolet Radiation, Thermal Cycling and Atomic Oxygen on Altered and Coated Kapton Surfaces
}

Joyce A. Dever

Lewis Research Center

Cleveland, Ohio

and

Eric J. Bruckner and Elvin Rodriguez

Cleveland State University

Cleveland, Ohio

Prepared for the

30th Aerospace Sciences Meeting and Exhibit

sponsored by the American Institute of Aeronautics and Astronautics

Reno, Nevada, January 6-9, 1992 


\title{
SYNERGISTIC EFFECTS OF ULTRAVIOLET RADIATION, THERMAL CYCLING AND ATOMIC OXYGEN ON ALTERED AND COATED KAPTON SURFACES
}

\author{
JOYCE A. DEVER* \\ NASA Lewis Research Center \\ Cleveland, $\mathrm{OH} 44135$ \\ ERIC J. BRUCKNER \\ ELVIN RODRIGUEZ \\ Cleveland State University \\ Cleveland, $\mathrm{OH} 44115$
}

\begin{abstract}
The photovoltaic (PV) power system for Space Station Freedom (SSF) utilizes solar array blankets which provide structural support for the solar cells and house the electrical interconnections. In the low Earth orbital (LEO) environment where SSF will be located, surfaces will be exposed to potentially damaging environmental conditions including solar ultraviolet (UV) radiation, thermal cycling and atomic oxygen. It is necessary to utilize ground-based tests to determine how these environmental conditions would affect the mass loss and optical properties of candidate SSF blanket materials. Silicone-containing, siliconecoated and $\mathrm{SiO}_{x}$ coated polyimide film materials were exposed to simulated LEO environmental conditions to determine their durability and whether the environmental conditions of UV, thermal cycling and atomic oxygen act synergistically on these materials. A candidate PV blanket material called AOR Kapton, a polysiloxane-polyimide cast from a solution mixture, shows an improvement in durability to atomic oxygen erosion after exposure to ultraviolet radiation or thermal cycling combined with ultraviolet radiation. This may indicate that the environmental conditions react synergistically with this material, and the

\footnotetext{
Member AIAA
}

Materials Engineer
\end{abstract}

damage predicted by exposure to atomic oxygen alone is more severe than that which would occur in LEO where atomic oxygen, thermal cycling and ultraviolet radiation are present together.

\section{INTRODUCTION}

The Space Station Freedom (SSF) photovoltaic (PV) power system will rely upon a durable blanket material to support the solar cells, house the electrical interconnections, and maintain the proper operating temperature for the solar arrays over its desired fifteen year lifetime. In low Earth orbit (LEO) the PV array blanket will experience 80,000 to 84,000 hours of solar ultraviolet (UV) radiation, 83,000 to 87,000 temperature cycles between approximately $+80^{\circ} \mathrm{C}$ and $-80^{\circ} \mathrm{C}$, and an atomic oxygen fluence of $4.85 \times 10^{22}$ atoms $/ \mathrm{cm}^{2}$ which is the orbital average for solar and antisolar facing array surfaces. ${ }^{1,2}$ It is important to determine the effects of these environmental conditions on candidate array blanket materials, because they are potentially threatening to their structural integrity and desired optical properties.

The material of choice for use on the PV array blankets is Kapton ${ }^{R}$, a polyimide material manufactured by DuPont, because of its desirable physical, optical and mechanical properties. By itself, however, Kapton will erode

Copyright ${ }^{\text {(2) }} 1992$ by the American Institute of Aeronautics and Astronautics, Inc. No copyright is asserted in the United States under Title 17, U.S. Code. The U.S. Government has a royalty-free license to exercise all rights under the copyright claimed herein for Governmental purposes. All other rights are reserved by the copyright owner. 
in the presence of atomic oxygen. In fact, a Kapton film thickness of $0.00254 \mathrm{~cm}(0.001 ")$ would be lost in only 0.26 years based on an erosion yield of $3 \times 10^{-24} \mathrm{~cm}^{3} /$ atom for Kapton in LEO ${ }^{3-8}$ and a flux of $1 \times 10^{14}$ atoms $/ \mathrm{cm}^{2} \mathrm{~s}$ which is the orbital average flux for solar and anti-solar facing surfaces. ${ }^{2}$ Such erosion would result in structural failure of the array blanket well in advance of its anticipated lifetime. Therefore, some protection or alteration of the Kapton is needed to improve its durability. The environmental conditions in LEO may also cause increases in solar absorptance of the PV array blanket material which would cause a temperature increase throughout the blanket. Less efficient operation of the solar cells would result. Therefore, it is necessary to be able to predict the changes in solar absorptance of candidate materials, and choose materials which are least susceptible to this type of damage.

Silicon dioxide is a material which can provide a barrier to oxidation of Kapton. ${ }^{9-12}$ The candidate PV array blanket materials evaluated in this study rely on this type of protection. These materials included Kapton coated with thin film $\mathrm{SiO}_{x}$ (where $\mathrm{x}$ is approximately 2), coatings of silicone on Kapton, and a siliconepolyimide film material. Reaction of silicone with atomic oxygen should result in formation of $\mathrm{SiO}_{2}$ at its surface and thus provide protection to the Kapton. ${ }^{12}$ The candidate materials studied include: $\mathrm{SiO}_{x}$ (x is between 1.9 and 2.0) coated Kapton manufactured by Sheldahl, Inc.; DuPont's AOR Kapton, a polysiloxane-polyimide film made from silicone and polyimide which is cast from a solution mixture; and MgGhan NuSil CV-1147 coated Kapton with or without a primer coating applied to the Kapton before application of the CV-1147. CV-1147 is a low-blocking silicone with a glass-frit filler.

Samples of candidate photovoltaic array blanket materials were exposed to vacuum ultraviolet (VUV) radiation (in the wavelength range between approximately 100 and $200 \mathrm{~nm}$ ) and vacuum thermal cycling combined with ultraviolet radiation (wavelength range between approximately 100 and $400 \mathrm{~nm}$ ) in separate tests. Changes in solar absorptance were determined. Samples exposed to these environments were then exposed in an RF air plasma asher, which simulates the atomic oxygen environment of LEO, to determine general atomic oxygen durability and whether the mass loss of samples exposed to the atomic oxygen environment alone was different than the mass loss of samples exposed to atomic oxygen after exposures to UV and UV/thermal cycling.

\section{LEO ENVIRONMENTAL SIMULATION FACILITIES AND PROCEDURES}

\section{Vacuum Ultraviolet Radiation Exposure}

Samples of Kapton coated with $\mathrm{SiO}_{x}$ or CV1147 silicone, a low-blocking silicone containing glass frit filler, were exposed to 1000 equivalent sun hours (ESH) of VUV. Samples of AOR Kapton were exposed to between 770 and $1160 \mathrm{ESH}$ VUV. The distance between the lamp and the samples was adjusted such that the lamp provided 5 VUV equivalent suns in the wavelength range between 100 and $200 \mathrm{~nm}$ for the $\mathrm{SiO}_{x}$ coated Kapton and AOR Kapton samples and 3 VUV equivalent suns for the CV1147 coated Kapton samples. The damage caused by 3 VUV suns and 5 VUV suns is not expected to be different for equivalent numbers of sun hours. System pressure for this testing was approximately $2 \times 10^{-6}$ torr.

\section{Vacuum Thermal Cycling Combined with Ultraviolet Radiation}

Samples of the $\mathrm{SiO}_{x}$ coated Kapton were exposed to 700 vacuum thermal cycles combined with approximately $700 \mathrm{ESH}$ VUV. The AOR Kapton material was exposed to 500 vacuum thermal cycles combined with approximately $500 \mathrm{ESH}$ VUV. The temperature limits for these exposures were between $-80^{\circ} \mathrm{C}$ and $+80^{\circ} \mathrm{C}$. The system pressure was below $10^{-6}$ torr. Lamps which were used in vacuum to provide VUV were two $30 \mathrm{~W}$ deuterium lamps with magnesium fluoride windows. These lamps provide VUV radiation between $118 \mathrm{~nm}$ and 200 $\mathrm{nm}$. Additionally a mercury-xenon short arc lamp was located outside the vacuum chamber. UV radiation between 200 and $400 \mathrm{~nm}$ was transmitted through a quartz window. The duration of each cycle, including exposure to heat and ultraviolet radiation followed by 
cooling, was approximately 26 minutes. The acceleration of VUV radiation during the heating portion of the cycle was 5 VUV equivalent suns between $118 \mathrm{~nm}$ and $200 \mathrm{~nm}$. The rate of heating was adjusted to bring the sample to its high temperature limit in one fifth the time it would take for the actual sun exposed portion of a Space Station orbit which is approximately 59 minutes. Therefore, during each test cycle, samples received the equivalent SSF orbit VUV dose in 11.8 minutes. The UV radiation in the wavelength range between 200 and $400 \mathrm{~nm}$ was not accelerated due to the large distance that was necessary between the sample and the lamp, because it was necessary to locate this lamp outside of the vacuum chamber. However, the UV lamp was used to provide representation of this range of wavelengths.

\section{Simulated Atomic Oxygen Exposure}

A plasma asher (SPI Plasma Prep II) was used to simulate the atomic oxygen environment of LEO. This instrument generates a $13.56 \mathrm{MHz}$ RF discharge of air containing various species of oxygen and nitrogen ions and atoms in a partial vacuum (60 to 100 mtorr). The air plasma asher exposure produces damage which is qualitatively similar to that which would occur in LEO, although there are significant differences in the exposure environments. The random directionality of attack observed in the air plasma asher produces undercutting which is broader and shallower than that predicted to occur due to the sweeping ram directionality of attack in space. $^{13}$ Additionally, the energy in the SSF environment in LEO is expected to be between 3.5 and $5.5 \mathrm{eV}^{12}$, whereas the asher energy is thermal, approximately $0.1 \mathrm{eV}$.

Samples which had been previously exposed to VUV radiation, vacuum thermal cycling combined with VUV radiation, and unexposed samples were then ashed in order to determine synergistic effects of VUV radiation, vacuum thermal cycling, and atomic oxygen. Because only one side of the material could be exposed in the VUV and thermal cycling/VUV facilities, but both sides of a sample were to be exposed to the plasma in the asher, sample pieces were clad together with acrylic adhesive such that the UV exposed sides of the materials were facing out.

Mass loss as a function of atomic oxygen effective fluence was determined for $\mathrm{SiO}_{x}$ coated Kapton, CV-1147 coated Kapton and AOR Kapton up to a total fluence of $5 \times 10^{21}$ atoms $/ \mathrm{cm}^{2}$.

\section{Optical Properties Analysis}

Transmittance and reflectance of samples were measured with a Perkin-Elmer Lambda-9 UV-VIS-NIR spectrophotometer with a $60 \mathrm{~mm}$ integrating sphere in the wavelength range between 250 and $2500 \mathrm{~nm}$. Solar absorptance $(\alpha)$ for non-opaque materials can be obtained as follows:

$$
\alpha=1-\tau-\rho
$$

where $\alpha$ is the solar absorptance, $\tau$ is the solar transmittance and $\rho$ is the solar reflectance. The solar reflectance, $\rho$, can be obtained from the values of spectral reflectance $\rho(\lambda)$ mathematically as follows ${ }^{14}$ :

$$
\rho=\frac{\int \rho(\lambda) S(\lambda) d \lambda}{\int S(\lambda) d \lambda}
$$

where the values of $S(\lambda)$ are the solar spectral irradiance values for air mass zero. The integrals are taken over the wavelength range between 250 and $2500 \mathrm{~nm}$. The solar transmittance, $\tau$, can be obtained using the same equation using values for spectral transmittance $\tau(\lambda)$ instead of spectral reflectance.

Solar absorptance values were obtained before and after VUV radiation exposure and atomic oxygen exposure. The error in solar absorptance values is estimated to be \pm 0.007 .

\section{RESULTS OF ENVIRONMENTAL EXPOSURE TESTING}

\section{$\underline{\mathrm{SiO}}_{x}$ Coated Kapton}

Solar Absorptance: 
The single layer sample of $\mathrm{SiO}_{x}$ coated Kapton exposed to 1000 ESH VUV showed a negligible change in solar absorptance from an initial value of 0.214 to a post-exposure value of 0.210 . $1000 \mathrm{ESH}$ represents approximately 68 days on SSF. The sample exposed to 700 vacuum thermal cycles containing VUV also showed a negligible change in solar absorptance from 0.206 to 0.209 . 700 cycles represents approximately 45 days on SSF. The resulting solar absorptance values are within the target solar absorptance $(\alpha \leq 0.22)$ for the photovoltaic array blanket materials at these levels of exposure.

Figure 1 shows the solar absorptance of acrylic adhesive clad samples of $\mathrm{SiO}_{x}$ coated Kapton as a function of air plasma ashing up to an effective atomic oxygen fluence of $3.7 \times 10^{20}$ atoms $/ \mathrm{cm}^{2}$. This level of exposure represents approximately 84 days on SSF. This plot shows that solar absorptance degradation is not enhanced by pre-exposure to VUV radiation or to vacuum thermal cycling combined with VUV radiation. In fact, at these levels of exposure, the solar absorptance of this material is relatively stable.

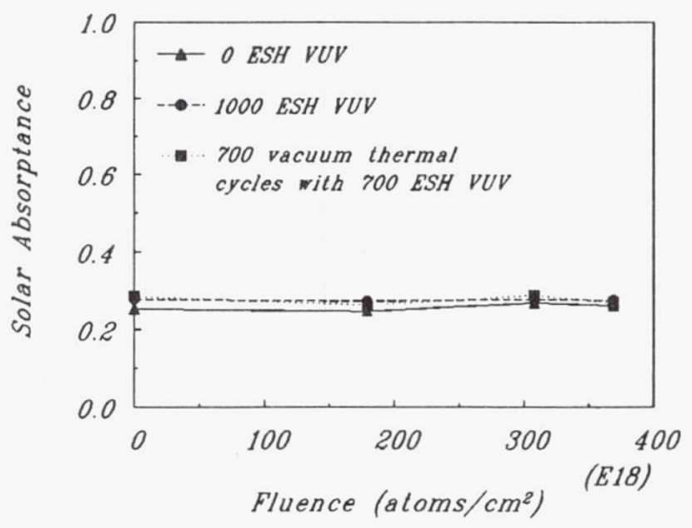

Figure 1: Effect of air plasma ashing on solar absorptance of $\mathrm{SiO}_{x}$ coated Kapton

Mass Loss:

A plot of mass loss as a function of effective atomic oxygen fluence upon air plasma ashing up to $3.7 \times 10^{20}$ atoms $/ \mathrm{cm}^{2}$ is shown in Figure 2. The plot shows that previous exposure to VUV or to vacuum thermal cycling combined with VUV did not significantly affect the mass loss rate over that of a sample which was not previously exposed for this fluence level.

\section{CV-1147 Coated Kapton}

Solar Absorptance:

A sample of CV1147 coated Kapton, with no primer coating on the Kapton,

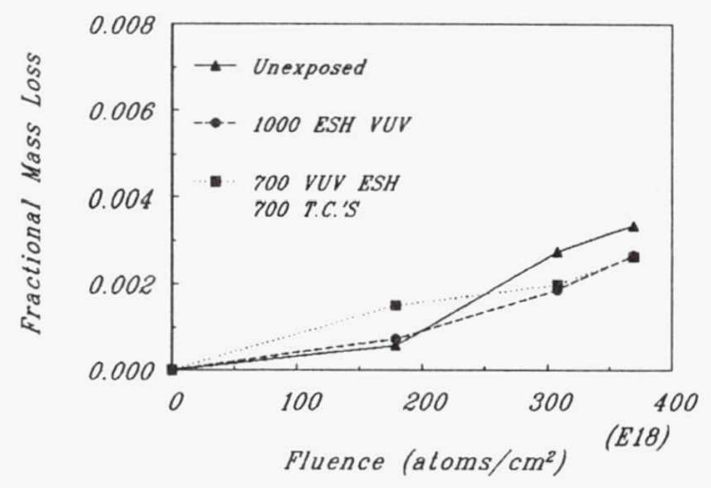

Figure 2: Effect of air plasma ashing on $\mathrm{SiO}_{x}$ coated Kapton

showed an increase in solar absorptance of 0.033 from 0.235 to 0.268 upon exposure to 1000 ESH VUV. The sample of CV1147 coated Kapton, with a primer coating on the Kapton, showed an increase in solar absorptance of 0.038 from 0.239 to 0.277 . These increases of $14-16 \%$ result in values which exceed the target $\alpha$ and indicate a potential instability in the solar absorptance of this material.

Figure 3 shows solar absorptance as a function of air plasma fluence for VUV exposed and unexposed CV1147 coated Kapton. Results indicate that the solar absorptance of nonprimed CV1147 coated Kapton which had been exposed to VUV radiation increased slightly more rapidly than the primed CV1147 coated Kapton which had been VUV exposed and the unexposed CV1147 coated Kapton samples.

\section{Mass Loss:}

Figure 4 shows the effects of air plasma ashing on the fractional mass loss of CV1147 coated Kapton. At this level of fluence, UV 
radiation exposure does not appear to effect the atomic oxygen damage, and fractional mass loss is minimal.

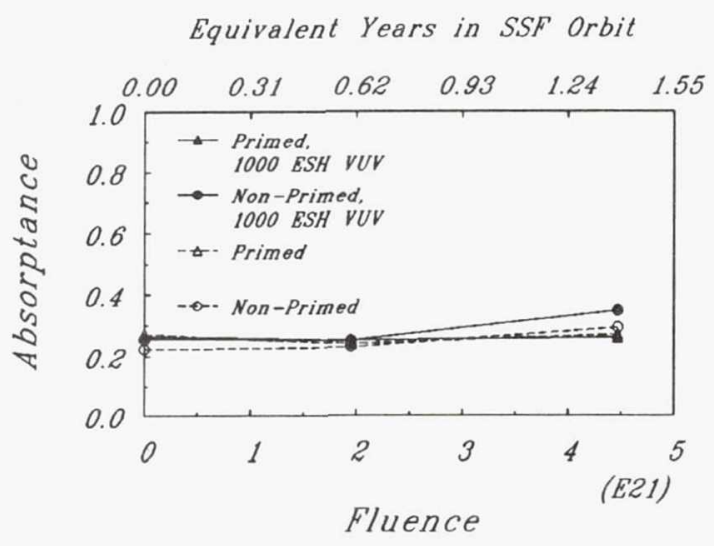

Figure 3: Effect of air plasma ashing on solar absorptance of CV-1147 coated Kapton

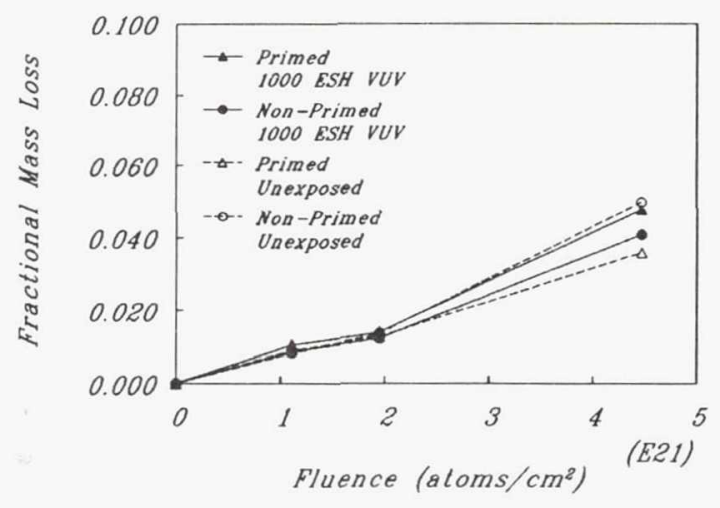

Figure 4: Effect of air plasma ashing on CV-1147 coated Kapton

\section{AOR Kapton}

\section{Solar Absorptance:}

The solar absorptance of AOR Kapton increased significantly upon exposure to VUV radiation as shown in Figure 5. This figure also shows that AOR Kapton displays the most severe solar absorptance degradation of all the candidate materials evaluated.

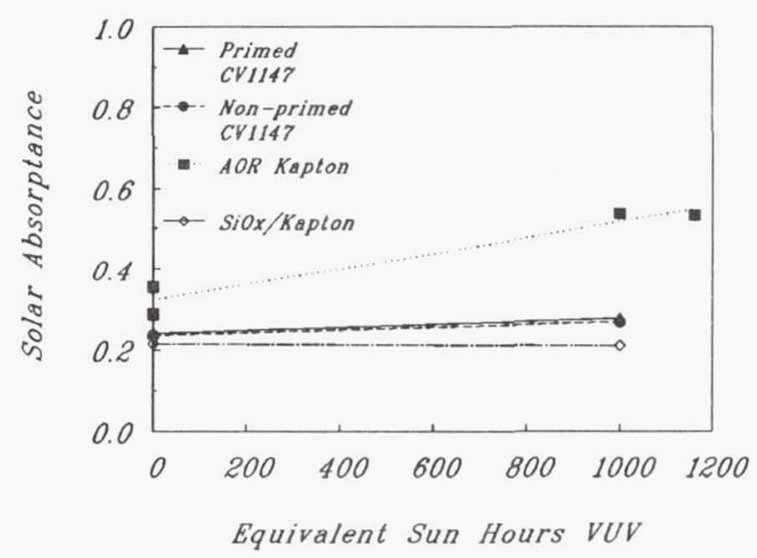

Figure 5: Effect of VUV radiation on solar absorptance

Mass Loss:

Figure 6 shows fractional mass loss of AOR Kapton as a function of air plasma ashing for atomic oxygen fluence up to $5 \times 10^{21}$ atoms $/ \mathrm{cm}^{2}$. These results show that samples which were previously exposed to VUV radiation or vacuum thermal cycling combined with VUV radiation degrade at a slower rate than samples which were not previously exposed. This indicates that the VUV, thermal cycling and atomic oxygen act synergistically on AOR Kapton. Samples exposed to thermal cycling combined with VUV radiation showed the lowest atomic oxygen mass loss of all the AOR Kapton samples studied. This may indicate that a combination of VUV radiation and high temperature affect the silicone-polyimide to significantly improve its atomic oxygen resistance. However, in comparing the durability of AOR Kapton to that of the other materials tested, even the thermal cycled/VUV exposed materials display atomic oxygen degradation which is considerably worse than that of the $\mathrm{SiO}_{x}$ or CV-1147 coated Kapton.

Scanning Electron Microscopy Results: Visual observation of the pristine AOR Kapton material reveals regions resembling "water spots" which probably occurred due to the use of a release-agent during the material processing.

In observing scanning electron micrographs of AOR Kapton exposed to an 


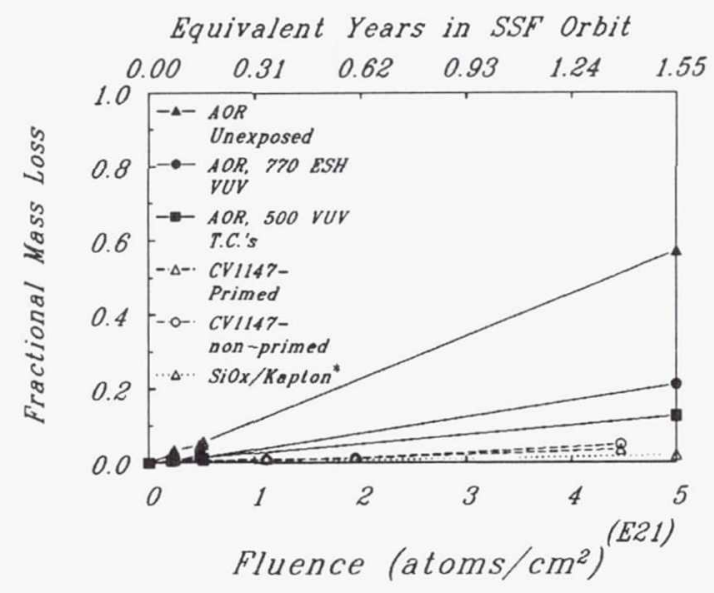

Figure 6: Air plasma ashing of candidate PV blanket materials (* Provided by Sharon Rutledge, NASA Lewis Research Center)

atomic oxygen fluence of $5 \times 10^{20}$ atoms $/ \mathrm{cm}^{2}$, two types of regions, bulk and water spot regions can be distinguished. The scanning electron micrograph in Figure 7a shows both types of regions. The region labeled " $\mathrm{A}$ " on the lower left is from the bulk area of the material. The region on the upper right labelled " $\mathrm{B}$ " is from the water spot region of the material. The bulk region is shown at a higher magnification in Figure $7 \mathrm{~b}$. The surface seems to be greatly attacked by atomic oxygen as evidenced by the perforations throughout the surface. Figure $7 \mathrm{c}$ shows a higher magnification electron micrograph of the water spot region. As shown, many nearly-spherical shaped particles exist in this region, and the surface does not show evidence of atomic oxygen degradation. Apparently, the processing of the AOR Kapton material results in differing chemistry between the bulk and water spot regions, and, as a result, the susceptibility to atomic oxygen degradation is different between these two regions.

Figure 8 is a micrograph showing a sample exposed to 770 ESH VUV which had then been exposed to $5 \times 10^{20}$ atoms $/ \mathrm{cm}^{2}$ atomic oxygen fluence. The micrograph shows a representative area of the sample which depicts that the bulk and water spot regions are relatively indistinguishable. Pinhole defects are shown which allowed atomic oxygen undercutting and resulted in formation of microscopic craters. However, the extent of damage is considerably less than that shown by the sample which received exposure to atomic oxygen without prior exposure to VUV (Figure $7 a$ and $b$ ). Also note, in Figure 8, the spherical shaped particles which are similar to those which were observed in the water spot region of the atomic oxygen exposed sample (Figure 7a and $\mathrm{C}$ ).

Figure 9a is a scanning electron micrograph of a sample exposed to 500 vacuum thermal cycles with approximately 500 ESH VUV followed by exposure to $5 \times 10^{20}$ atoms $/ \mathrm{cm}^{2}$ fluence of atomic oxygen. Again, it is difficult to distinguish between the bulk and the water spot regions. This sample also had pinhole defects (Figure 9b) throughout the surface which were attacked and undercut by atomic oxygen. However, the damage to this material was, as in the case of the sample previously exposed to VUV, not nearly as extensive as the damage to the bulk area of the sample exposed only to atomic oxygen (Figure $7 a$ and b).

\section{DISCUSSION}

\section{Solar Absorptance}

As shown in Figure 5, the AOR Kapton shows the greatest degradation in solar absorptance upon exposure to VUV radiation. This degradation is considered unacceptable to the requirements of the SSF photovoltaic array blanket, because a low (target $\alpha \leq 0.22$ ) and long-term stable solar absorptance is required. Such increases in solar absorptance of the solar array blanket material will cause a temperature increase in the solar cells mounted to the blanket resulting in less efficient operation of the system. Both the primed and non-primed CV1147 coated Kapton materials show significantly lower initial values of solar absorptance and their values remain much more stable than AOR Kapton, despite a $14-16 \%$ increase. The $\mathrm{SiO}_{x}$ coated Kapton showed the lowest values and greatest stability in solar absorptance. 


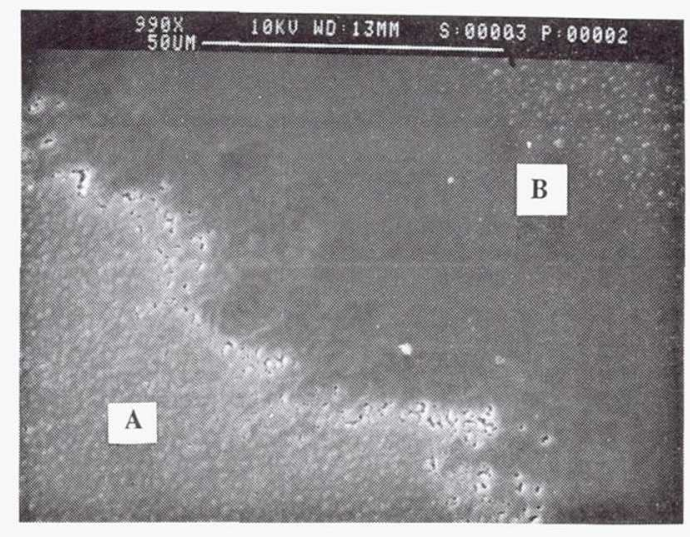

a) Bulk and water spot regions

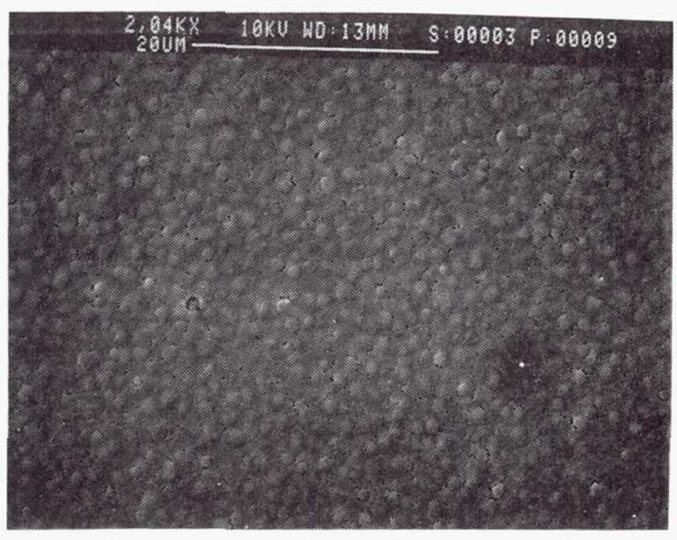

b) Bulk region

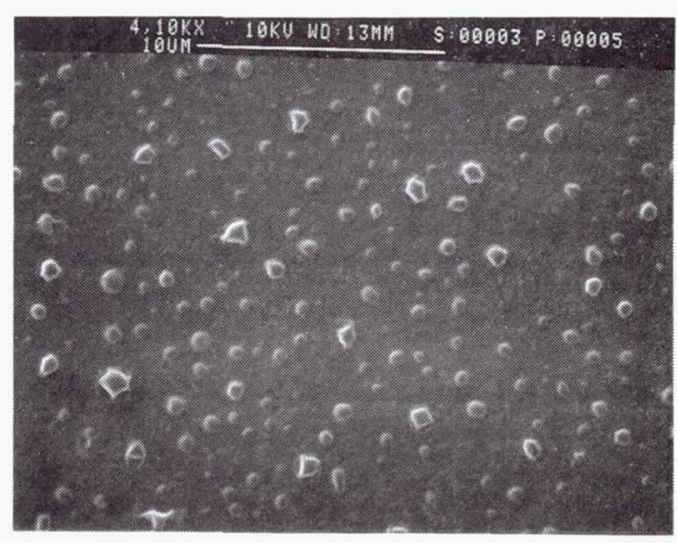

c) Water spot region

Figure 7: Air plasma ashed AOR Kapton

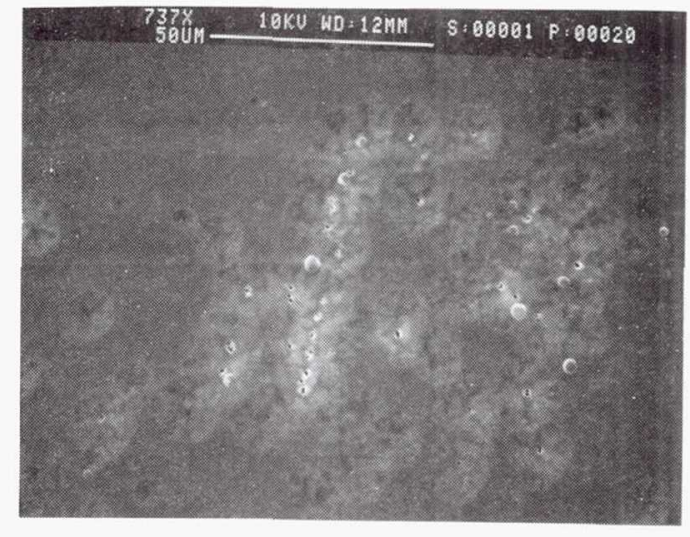

Figure 8: AOR Kapton exposed to VUV followed by air plasma ashing

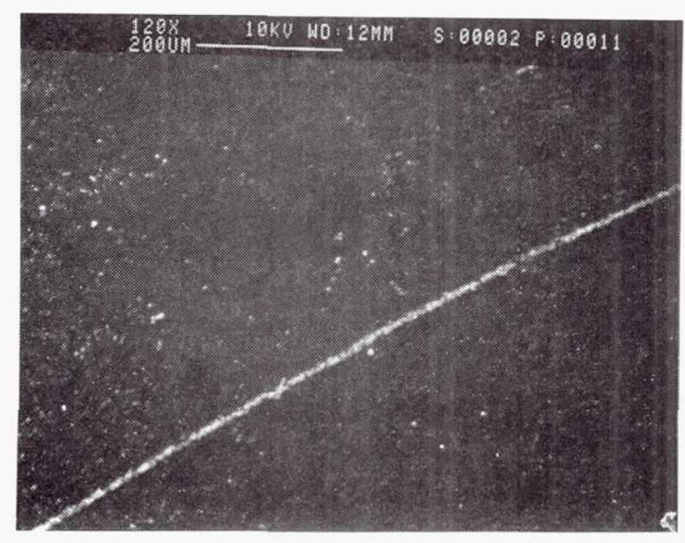

a) Surface defects

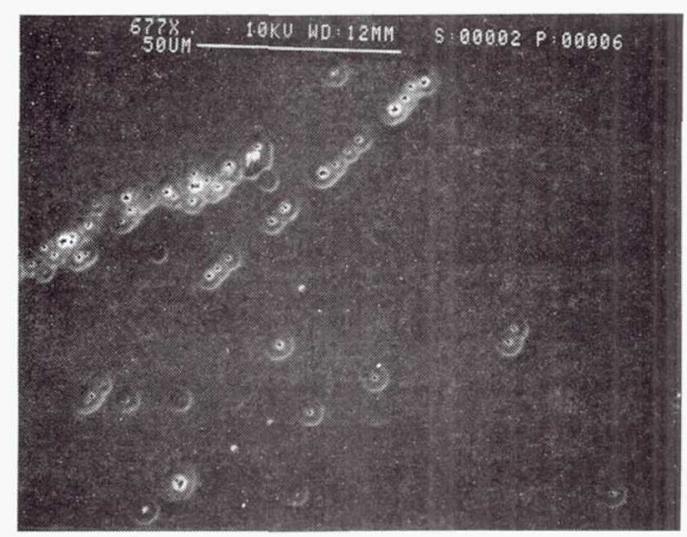

b) Undercutting of pinhole defects

Figure 9: AOR Kapton exposed to vacuum thermal cycling with VUV followed by air plasma ashing 
Neither the $\mathrm{SiO}_{x}$ coated Kapton, nor the primed CV1147 coated Kapton, showed a significant change in susceptibility to degradation of solar absorptance upon air plasma ashing as a result of previous exposure to VUV radiation or vacuum thermal cycling combined with VUV radiation (Figures 1 and 3). Therefore, VUV and thermal cycling do not act synergistically with atomic oxygen to affect the mass loss of these materials. The non-primed CV1147 coated Kapton which had been exposed to 1000 ESH VUV showed a slightly greater degradation in solar absorptance than VUV exposed, primed samples and the unexposed, primed or non-primed samples as shown in Figure 3. At this level of exposure, representative of 1.55 years in SSF orbit, the solar absorptance of the VUV exposed nonprimed material shows degradation which significantly exceeds the target solar absorptance.

\section{Mass Loss}

The $\mathrm{SiO}_{x}$ coated Kapton and the CV1147 coated Kapton displayed significantly less fractional mass loss than the AOR Kapton material when exposed to a level of fluence equivalent to 1.55 years in SSF orbit (Figure 6). Additionally, CV1147 and $\mathrm{SiO}_{x}$ coated Kapton do not show a difference in susceptibility to atomic oxygen erosion due to previous exposure to VUV or thermal cycling combined with VUV (Figures 2 and 4). However, it was observed that the AOR Kapton becomes less susceptible to atomic oxygen attack as a result of previous exposure to VUV radiation and vacuum thermal cycling combined with VUV. This suggests that VUV radiation reacts with this silicone-polyimide material to improve its resistance to atomic oxygen attack. Therefore, VUV, thermal cycling and atomic oxygen react synergistically with AOR Kapton. Because of the significance of the difference in mass loss upon air plasma ashing between the AOR Kapton samples which were previously exposed to environments containing VUV and samples of unexposed AOR Kapton materials, it is important to consider that the mass loss predicted by atomic oxygen exposure alone may be excessive compared to what would actually occur in LEO where solar ultraviolet radiation is present along with atomic oxygen.

\section{CONCLUSIONS}

As evidenced by the example of polysiloxane-polyimide AOR Kapton, which shows an improvement in durability to atomic oxygen erosion after exposure to environments containing VUV radiation, it is important to determine the synergistic effects of solar ultraviolet (UV) radiation, thermal cycling, and atomic oxygen to assess the overall LEO environmental effects of LEO on materials. Results for the durability testing of the AOR Kapton material indicate that the damage predicted by exposure to atomic oxygen alone may be more severe than that which would occur in LEO where exposure atomic oxygen, ultraviolet radiation and thermal cycling occur simultaneously and have the opportunity to act synergistically. Synergistic effects as observed for AOR Kapton are not observed on all types of silicone materials, however. For example, coatings of CV1147 low-blocking silicone with and without glass frit-filler, did not show a difference in susceptibility to atomic oxygen damage upon exposure to environments containing VUV or thermal cycling. AOR Kapton and CV-1147 coated Kapton showed initial values of solar absorptance or increases in solar absorptance which exceed the target $\alpha$ of $\leq$ 0.22 . Of the three types of candidate SSF photovoltaic blanket materials discussed in this paper, $\mathrm{SiO}_{\times}$coated Kapton shows the best durability in terms of solar absorptance and atomic oxygen erosion.

\section{REFERENCES}

1. J. Brady and B. Banks, "Vacuum Ultraviolet Radiation and Thermal Cycling Effects on Atomic Oxygen Protective Photovoltaic Array Blanket Materials," in Materials Degradation in Low Earth Orbit, V. Srinivasan and B. A. Banks, eds., The Minerals, Metals and Materials Society, 1990, pp. 133-143.

2. Specification Number RC1800, Revision 
C, Rockwell International Corporation, Rocketdyne Division, Canoga Park, CA, January 11, 1990, p. 18.

3. L. G. Leger, B. Santos-Mason, J. T. Visentine, and J. F. Kuminecz, "Review of LEO Flight Experiments,"

Proceedings of NASA Workshop on Atomic Oxygen Effects, November, 1986, p. 6.

$4 . \quad$ L. J. Leger, I. K. Spiker, J. F. Kuminecz, T. J. Ballentine and J. T. Visentine, "STS Flight 5 LEO Effects Experiment Background Description and Thin Film Results," AIAA-83-2631-CP, October, 1983.

5. L. J. Leger, "Oxygen Atom Reaction with Shuttle Materials at Orbital Altitudes - Data and Experiment Status," AIAA-830073, January 1983.

6. B. A. Banks, M. J. Mirtich, S. K. Rutledge, H. K. Nahra, "Protection of Solar Array Blankets from Attack by Low Earth Orbital Atomic Oxygen," 18th IEEE Photovoltaic Specialists Conference, October, 1985.

7. J. T. Visentine, L. G. Leger, J. F. Kuminecz, and I. K. Spiker, "NASA JSC STS-8 Atomic Oxygen Effects Experiment," AIAA 23rd Aerospace Sciences Meeting, January 1985.

8. D. R. Coulter, R. H. Liang, S. H. Chung, K. O. Smith and A. Gupta, "O-atom Degradation Mechanisms of Materials," from Proceedings of NASA Workshop on Atomic Oxygen Effects, June 1, 1987, p. 42.

9. S. Rutledge, et al, "An Evaluation of Candidate Oxidation Resistant Materials for Space Applications in LEO," NASA Technical Memorandum 100122, November 1986.

10. B. A. Banks, et al, "Ion Beam SputterDeposited Thin Film Coatings for Protection of Spacecraft Polymers in
Low Earth Orbit," NASA Technical Memorandum 87051, January, 1985.

11. B. A. Banks, et al, "Sputtered Coatings for Protection of Spacecraft Polymers," NASA Technical Memorandum 83706, April 1984.

12. S. K. Rutledge and J. A. Mihelcic, "The Effect of Atomic Oxygen on Altered and Coated Kapton Surfaces for Spacecraft Applications in Low Earth Orbit," in Materials Degradation in Low Earth Orbit, V. Srinivasan and B. A. Banks, eds., The Minerals, Metals and Materials Society, 1990, pp. 35-48.

13. B. A. Banks et al, "Atomic Oxygen Undercutting of Defects on $\mathrm{SiO}_{2}$ Protected Polyimide Solar Array Blankets," in Materials Degradation in Low Earth Orbit, V. Srinivasan and B. A. Banks, eds., The Minerals, Metals and Materials Society, 1990, pp. 15-33.

14. J. H. Henninger, "Solar Absorptance and Thermal Emittance of Some Common Spacecraft Thermal-Control Coatings," NASA Reference Publication 1121, April 1984. 
Public reporting burden for this collection of information is estimated to average 1 hour per response, including the time for reviewing instructions, searching existing data sources, gathering and maintaining the data needed, and completing and reviewing the collection of information. Send comments regarding this burden estimate or any other aspect of this collection of information, including suggestions for reducing this burden, to Washington Headquarters Services, Directorate for information Operations and Reports, 1215 Jefferson Davis Highway, Suite 1204, Arlington, VA 22202-4302, and to the Office of Management and Budget, Paperwork Reduction Project (0704-0188), Washington, DC 20503.

\begin{tabular}{|l|c|r|r|}
\hline 1. AGENCY USE ONLY (Leave blank) & $\begin{array}{r}\text { 2. REPORT DATE } \\
1991\end{array}$ & $\begin{array}{r}\text { 3. REPORT TYPE AND DATES COVERED } \\
\text { Technical Memorandum }\end{array}$ \\
\hline
\end{tabular}

\section{TITLE AND SUBTITLE}

5. FUNDING NUMBERS

Synergistic Effects of Ultraviolet Radiation, Thermal Cycling and Atomic

Oxygen on Altered and Coated Kapton Surfaces

6. AUTHOR(S)

Joyce A. Dever, Eric J. Bruckner, and Elvin Rodriguez

$$
\text { WU-474-46-10 }
$$

7. PERFORMING ORGANIZATION NAME(S) AND ADDRESS(ES)

8. PERFORMING ORGANIZATION REPORT NUMBER

National Aeronautics and Space Administration

Lewis Research Center

Cleveland, Ohio 44135-3191

E- 6741

9. SPONSORING/MONITORING AGENCY NAMES(S) AND ADDRESS(ES)

10. SPONSORING/MONITORING AGENCY REPORT NUMBER

National Aeronautics and Space Administration

Washington, D.C. 20546-0001

NASA TM- 105363

AIAA-92-0794

\section{SUPPLEMENTARY NOTES}

Prepared for the 30th Aerospace Sciences Meeting and Exhibit sponsored by the American Institute of Aeronautics and Astronautics Reno, Nevada, January 6 - 9, 1992. Joyce A. Dever, NASA Lewis Research Center, Eric J. Bruckner and Elvin Rodriguez, Cleveland, State University, Cleveland, Ohio 44115. Responsible person, Joyce A. Dever, (216) 433-6294.

12a. DISTRIBUTION/AVAILABILITY STATEMENT 12b. DISTRIBUTION CODE

Unclassified - Unlimited

Subject Category 23

\section{ABSTRACT (Maximum 200 words)}

The photovoltaic (PV) power system for Space Station Freedom (SSF) utilizes solar array blankets which provide structural support for the solar cells and house the electrical interconnections. In the low Earth orbital (LEO) environment where SSF will be located, surfaces will be exposed to potentially damaging environmental conditions including solar ultraviolet (UV) radiation, thermal cycling and atomic oxygen. It is necessary to utilize ground-based tests to determine how these environmental conditions would affect the mass loss and optical properties of candidate SSF blanket materials. Silicone-containing, silicone-coated and $\mathrm{SiO}_{\mathrm{x}}$ coated polyimide film materials were exposed to simulated LEO environmental conditions to determine their durability and whether the environmental conditions of UV, thermal cycling and atomic oxygen act synergistically on these materials. A candidate PV blanket material called AOR Kapton, a polysiloxane-polyimide cast from a solution mixture, shows an improvement in durability to atomic oxygen erosion after exposure to ultraviolet radiation or thermal cycling combined with ultraviolet radiation. This may indicate that the environmental conditions react synergistically with this material, and the damage predicted by exposure to atomic oxygen alone is more severe than that which would occur in LEO where atomic oxygen, thermal cycling and ultraviolet radiation are present together.

\section{SUBJECT TERMS}

Ultraviolet radiation; Oxygen atoms; Space environment simulation; Protective coatings

\begin{tabular}{l|c} 
17. SECURITY CLASSIFICATION & $\begin{array}{c}\text { 18. SECURITY CLASSIFICATION } \\
\text { OF REPORT } \\
\text { OF THIS PAGE } \\
\text { Unclassified }\end{array}$
\end{tabular}

\section{SECURITY CLASSIFICATION OF ABSTRACT Unclassified}

